\title{
Some Results on FGS-modules
}

\author{
ALhousseynou BA ${ }^{1}$, Albert Mankagna Diompy ${ }^{1}$, Alassane Diouf ${ }^{1} \&$ André Souleye Diabang ${ }^{1}$ \\ ${ }^{1}$ Department of Mathematics and Computer Sciences, Cheikh Anta Diop University, Dakar, Senegal \\ Correspondence: Alhousseynou BA, Department of Mathematics and Computer Sciences, Faculty of Sciences and Tech- \\ mics, Cheikh Anta Diop University, B.P. 5005, - Dakar-Fann, - Senegal
}

Received: October 12, 2016 Accepted: December 5, 2016 Online Published: December 30, 2016

doi:10.5539/jmr.v9n1p36

URL: http://dx.doi.org/10.5539/jmr.v9n1p36

\begin{abstract}
Let $R$ be a commutative ring, with a unity $1 \neq 0$ and $M$ a unitary left $R$-module. In this paper we give some properties of an $F G S$-module. After that we give others important characterizations. Indeed, we first show that $M$ is a local $F G S$-module if and only if it is of finite representation type. Secondly, we show that $M$ is a prime $F G S$-module if and only if it is a serial type module and of finite length if and only if it is a finite representation type module.
\end{abstract}

Keywords: Hopfian, finitely generated, finite representation type, local

\section{Introduction}

Let $R$ be a commutative ring with $1 \neq 0$ as unity and $M$ a left module over $R$. The category $\sigma[M]$ is a full subcategory of $R$-Mod. Its objects are all submodules of a $M$-generated module(Wisbauer, R., 1985). we call that a module $M$ is Hopfian if every epimorphism of $M$ is an automorphism of $M$. For a commutative ring any finitely generated module is Hopfian but Hopfian module is not always finitely generated(Ba, A. \& Diankha, O., 2013). Therefore we characterize the modules for which every Hopfian object of $\sigma[M]$ is finitely generated. These modules are called $F G S$-modules.

An object $N$ of $\sigma[M]$ is said to be coherent if it is finitely generated and every finitely generated submodule of $K$ is finitely presented. If any submodule of a module $M$ is an intersection of maximal submodule then, $M$ is called co-semismple. A module $M$ is said $\operatorname{good}$ if $M / \operatorname{Rad}(M)$ is co-semisimple where $\operatorname{Rad}(M)$ is the Jacobson radical of $M$. A module is uniserial if its submodules are linearly ordered by inclusion. A module is said serial (resp. semisimple) if it is direct sum of uniserial(resp. simple) modules. A module is said serial type if every object of $\sigma[M]$ is direct sum of uniserial modules of finite length. A module $M$ is said to be prime module if for any submodule $N$ of $M$, we have $\operatorname{Ann}(N)=\operatorname{Ann}(M)$. A module of finite length is finite representation type if there exists, in $\sigma[M]$, only many non-isomorphic finitely generated indecomposable modules. A ring $R$ is said to be $S$-ring if any Hopfian module over $R$ is noetherian.

\section{Some Properties of $F G S$-module}

In this part we give some preliminaries results which we will use in this paper.

Proposition 1 Let $R$ be a commutative ring and $M$ a finitely generated prime FGS-module. Then, $M$ is simple.

Proof. Since $M$ is finitely generated.(Wisbauer, R., 1991) that $\sigma[M]=R / A n n(M)$-Mod i.e any object of $\sigma[M]$ is a module over $R / A n n(M)$. As $M$ is an $F G S$-module then $R / A n n(M)$ is also an $F G S$-ring. It results from(Gueye, C. T. \& Sangharé, M.,2004)that $R / \operatorname{Ann}(M)$ is an artinian ring. We know that any finitely generated module over an artinian ring is artinian. Hence, $M$ is an artinian module. Therefore, there exists a minimal submodule in $M$. Let $N_{1}$ be that minimal submodule and the following diagram:

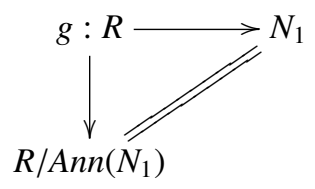

We can see that $R / \operatorname{Ann}\left(N_{1}\right) \simeq N_{1}$. Hence, $R / \operatorname{Ann}\left(N_{1}\right)$ is a field.

Let $g: R \longrightarrow M$ be an epimorphism. Therefore, $M \simeq R / \operatorname{Ann}(M)$. As $M$ is a prime module( i.e $\operatorname{Ann}(M)=\operatorname{Ann}\left(N_{1}\right)$ ), then $M$ is simple.

Corollary 2 Let $R$ be a commutative ring and $M$ a finitely generated, faithful and prime FGS-module then, $R$ is a field.

Proof. We have seen in proposition 1 that $R / \operatorname{Ann}\left(N_{1}\right)$ is simple. Since $M$ is prime then, $R / \operatorname{Ann}\left(N_{1}\right)=R / \operatorname{Ann}(M)$ is simple. As $M$ is faithful then, $R$ is a field.

Proposition 2 Let $R$ be a commutative ring and $M$ a prime and finitely generated FGS -module. Then: 
(1) Every submodule of an object $N$ of $\sigma[M]$ is maximal;

(2) There exists a finite number of submodules in $N$.

Proof. (1) It follows from the proposition 1 that $M$ is simple, hence semisimple. Therefore, every module of $\sigma[M]$ is semisimple (Wisbauer, R., 1991). Let $N$ be an objet of $\sigma[M]$ and $\left\{K_{j}\right\}_{J}$ a family of submodules of $N$. Let's assume $L=\bigoplus_{j \in J} N / K_{j} . L \in \sigma[M]$ because $\sigma[M]$ is closed under direct sum. As, any object of $\sigma[M]$ is semisimple then, $L$ is semisimple too. Hence, for every $j \in J, N / K_{j}$ is simple.Thus, for every $j \in J, K_{j}$ is maximal.

(2) Let's suppose $L=\bigoplus_{j \in J} N / K_{j}$. We have already seen that for any $j \in J N / K_{j}$ is simple. And it is obvious to see that, for all $j \in J, N / K_{j}$ is Hopfian and fully invariant. Then $L=\bigoplus_{j \in J} N / K_{j}$ is Hopfian. As $M$ is an $F G S$-module, then $L$ is finitely generated. Thus, $J$ is finite.

Lemma 1 If $M$ is a local module then, $M$ is finitely generated.

Proof. It follows from 21.6 of (Wisbauer, R., 1991) and the definition of local module.

Proposition 3 Let $R$ be commutative ring and $M$ a local $F G S$-module. Then, for every object $N$ of $\sigma[M]$, the following statements are equivalent:

(a) $N$ is finitely generated;

(b) $N$ is noetherian;

(c) $N$ is artinian;

(d) $N$ is of finite length.

Proof. Let $M$ be a local module. By lemma $1, M$ is finitely generated. Hence $\sigma[M]=R / A n n(M)$-Mod i.e every object of $\sigma[M]$ is a $R / \operatorname{Ann}(M)$-module. Since, $M$ is an $F G S$-module then, $R / A n n(M)$ is an $F G S$-ring. Hence, $R / A n n(M)$ is an artinian ring. It results from 15.21 of (Anderson, F. W. \& Fuller, K., 1973) that $(a),(b),(c)$ and $(d)$ are equivalent.

Lemma 2 (Anderson, F.W \& Fuller, K., 1973) $R$ is noetherian iff every finitely generated $R$-module is finitely presented.

Proposition 4 Let $M$ be a local $F G S$-module. Then, $M$ is a coherent module in $\sigma[M]$.

Proof. We have already seen that $M$ is finitely generated. Hence, $M \simeq R / A n n(M)$. It results from the proposition 1 that $R / \operatorname{Ann}(M)$ is artinian. It is well known that any artinian ring is noetherian, hence $R / \operatorname{Ann}(M)$ is a noetherian ring. Let $N$ be a finitely generated submodule of $M . N$ is also module over $R / \operatorname{Ann}(M)$. It follows from the lemma 2 that $N$ is finitely presented. Thus, $M$ is coherent.

Proposition 5 Let $M$ be a local FGS-module, then $M$ is a good module and so is every module of $\sigma[M]$.

Proof. As $M$ is local then, $M / \operatorname{Rad}(M)$ is simple hence semisimple. It is well know that any semisimple module is cosemisimple, hence $M / \operatorname{Rad}(M)$ is co-semisimple. By referring to 23.3 of (Wisbauer, R., 1991), $M$ is a good module. Let $N$ be an object of $\sigma[M] . N$ is a module over $R / \operatorname{Ann}(M)$. We have seen that $M \simeq R / \operatorname{Ann}(M)$, hence $R / \operatorname{Ann}(M)$ is good ring. It results from 23.7 of (Wisbauer, R., 1991) that $N$ is a good module.

\section{Results}

Lemma 3 If $M$ is an FGS-module then, there exists a finite number of non-isomorphic simple modules in $\sigma[M]$.

Proof. It results from proposition 2 of (Ba, A. \& Diankha, O., 2013).

Theorem 1 Let $R$ be a commutative ring and $M$ a local module then, the following assertions are equivalent:

(1) $M$ is an FGS-module;

(2) $M$ is of finite representation type.

Proof. (1) $\Rightarrow$ (2) By the proof of proposition $1 M \simeq R / \operatorname{Ann}(M)$ is artinian. Since $M$ is of finitely generated, then is of finite length. It results from the lemma 3 that $M$ is of finite representation type.

(2) $\Rightarrow$ (1) We have already seen that $M \simeq R / \operatorname{Ann}(M)$. As $M$ is a finite representation type, then it is of finite length and it follows from theorem 3.1 of (Diankha, O., 2007) that $M$ is an $I$-module. Hence $R / A n n(M)$ is an $I$-ring. It results theorem 9 of (Kaidi, A. \& Sanghare, M., 1965) that $R / \operatorname{Ann}(M)$ is a $S$-ring.

Let $N$ be an Hopfian object of $\sigma[M]$. Since $R / \operatorname{Ann}(M)$ is $S$-ring then $N$ is noetherian. Any noetherian module of an artinian ring is finitely generated. Thus $M$ is an $F G S$-module.

Theorem 2 Let $R$ be a commutative ring and $M$ a prime module. Then, the following assertions are equivalent:

(1) $M$ is an FGS-module;

(2) $M$ is a serial type and of finite length;

(3) $M$ is of finite representation type. Proof. (1) $\Rightarrow$ (2) Assume that $M$ is an $F G S$-module. It follows from proposition 1 that $M$ is a simple module. Hence it of finite length and semisimple. Let $N=\oplus_{i \in I} N_{i}$ be an element of $\sigma[M]$. Since $N$ is a semisimple module, then $N_{i}$ is a simple module for all $i \in I$. It is uniserial and of finite length. Therefore $M$ is of serial 
type.

(2) $\Rightarrow$ (3) It follows from 55.14 of (Wisbauer, R., 1991) that $M$ is of finite representation type.

(3) $\Rightarrow$ (1) It results from theorem 1 .

\section{References}

Anderson, F. W, \& Fuller, K. (1973). Rings and categories of modules. Springer-Verlag.

Ba, A., \& Diankha, O. (2013). On FGS-Modules. Journal of Mathematics Research, 5(1), 61-64, ISSN 1916-9795.

Diankha, O. (2007). On I-modules. Journal des Sciences, 7, $N^{\circ} 2$.

Gueye, C. T., \& Sangharé, M. (2004). On commutative FGS-rings. Communications in Algebra volume, 32(5), 17151727.

Kaidi, A., \& Sanghare, M. (1988). Une caractérisation des anneaux artiniens á ideaux principaux. Lect. Note in Math. N?328 Springer-verlag,245-254.

Vanaja, N. (1996). All finitely generated $M$-subgenerated modules are extending. Comm. Algebra, $24(2), 543-572$.

Wisbauer, R. (1985). Decomposition properties in modules categories. Acta. Univ. Corilia Math. Physica, 126(26), 57-68.

Wisbauer, R. (1991). Foundation of Module and Ring theory. Gordon and Breach Science Publishers.

\section{Copyrights}

Copyright for this article is retained by the author(s), with first publication rights granted to the journal.

This is an open-access article distributed under the terms and conditions of the Creative Commons Attribution license (http://creativecommons.org/licenses/by/4.0/). 